\title{
The James Webb Space Telescope Integrated Science Instrument Module
}

\author{
Matthew A. Greenhouse*, Pamela C. Sullivan, Leslye A. Boyce, Stuart D. Glazer, Eric L. Johnson, \\ John C. McCloskey, Mark F. Voyton \\ Goddard Space Flight Center, Greenbelt, MD 20771
}

\begin{abstract}
The Integrated Science Instrument Module of the James Webb Space Telescope is described from a systems perspective with emphasis on unique and advanced technology aspects. The major subsystems of this flight element are described including: structure, thermal, command and data handling, and software.
\end{abstract}

Keywords: JWST, Instrumentation

\section{INTRODUCTION}

The James Webb Space Telescope (JWST) mission (Figure 1) is under development by NASA for launch during 2011 with major contributions from the European and Canadian Space Agencies. The JWST mission is designed to address four science themes: [1] observation of the first luminous objects after the Big Bang, [2] the assembly of these objects into galaxies, [3] the birth of stars and planetary systems, and [4] the formation of planets and the origins of life. The Integrated Science Instrument Module (ISIM) is the science payload of the JWST and is one of three elements that comprise the JWST flight segment (Figure 2). The ISIM contains four science instrument sub-systems, a cryogenic fine guidance sensor, and a wavefront sensor that is used to control the optical figure of the telescope primary mirror. The primary function of the ISIM is to provide

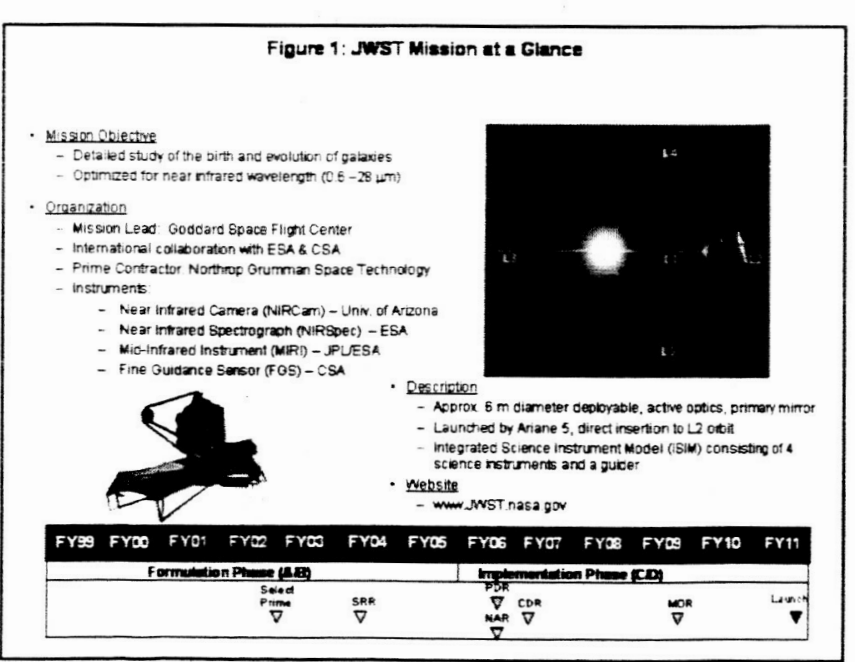
shared support systems for these instruments and to enable ground verification of the cryogenic payload prior to integration with the telescope. In

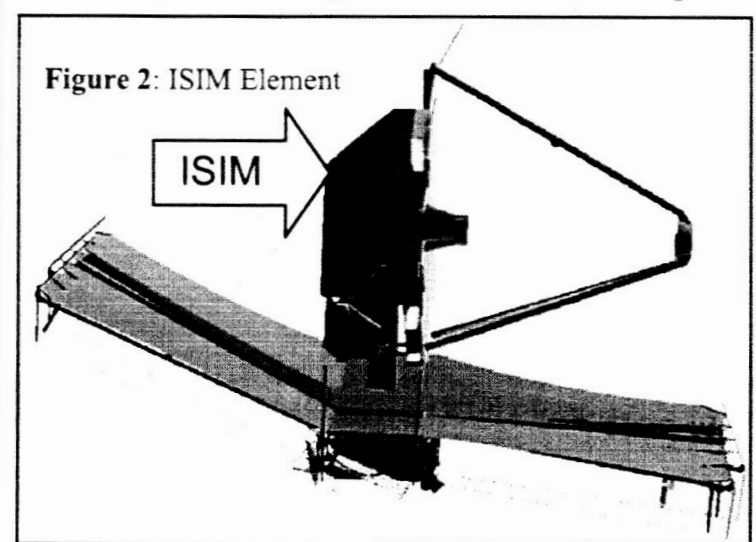

the following sections, we briefly describe the science instruments and their shared support systems including the optical metering structure, passive cryogenic thermal control, command and data handling (ICDH), and ICDH software. Further information on the JWST mission can be found at: http://jwst.gsfc.nasa.gov.

"matt.greenhouse@nasa.gov, 301 286-4517 


\section{SCIENCE INSTRUMENTATION}

\subsection{The Near-Infrared Camera (NIRCam)}

The functionality incorporated into the NIRCam design has changed only slightly in the last year. Table 1 summarizes the key elements of NIRCam's capabilities, which remain aimed at providing efficient surveying in two colors simultaneously and with a field as large as is commensurate with the number of pixels available. The optical design uses anti-reflection coated lenses and fold flats with the wavelength separation done using dichroics. The detector arrays for each wavelength regime in NIRCam have sensitivity ranges matched to the needs of the particular arm -- the short wavelength arm will use $\mathrm{HgCdTe}$ with a $2.5 \mu \mathrm{m}$ cut-off and will be thinned to support operation at as short a wavelength as $0.6 \mu \mathrm{m}$ and the long wavelength arm uses $\mathrm{HgCdTe}$ with a $5.1 \mu \mathrm{m}$ cut-off and no thinning. Using two types of detectors relaxes the requirements for out-of-band blocking for NIRCam's bandpass filters. Wavefront sensing needs are addressed by having pupil and filter wheels (Figure 3) with excellent repeatability in their positioning and by having redundant optical trains. The major new addition is a pupil imaging lens to aid in alignment and wavefront sensing. The pupil imaging will be done in the short wavelength arm of NIRCam seen on the right hand side of Figure 4 (pupil imaging lens is not shown). The other recent design change results from the selection of $\mathrm{HgCdTe}$ arrays, which allows the focal plane assemblies to be cooled through the instrument base plate rather than with dedicated radiators.

Many details of the design are being refined as the team works towards its preliminary design review including selection of cryogenic motors, lens mounts, and optical materials. For further information see S. Horner, et al.: paper 5487-25, of these proceedings.

\begin{tabular}{|c|c|}
\hline Wavelength Range & $0.6-5.0 \mu \mathrm{m}$ \\
\hline Optical Design & Refractive with dichroic splitting range into $0.6-2.4 \mu \mathrm{m}$ and $2.4-5.0 \mu \mathrm{m}$ \\
\hline Pixel Scales & $\begin{array}{l}0.0317 \% / \text { pixel (short wavelength ) } \\
0.0648 \% / \text { pixel (long wavelength) }\end{array}$ \\
\hline Coronagraphy & Focal plane and pupil masks available for both wavelength ranges \\
\hline Bench & Bonded $\mathrm{Be}$ \\
\hline Filter/Pupil Wheels & DC Direct Drive w/ position feedback \\
\hline Pick-off mirrors & Adjustable in focus \\
\hline $\begin{array}{l}\text { Detectors (40Mpixels } \\
\text { total) }\end{array}$ & $\begin{array}{l}4 \mathrm{Kx} 4 \mathrm{~K} \text { using } 2.5 \mu \mathrm{m} \text { cutoff } \mathrm{HgCdTe} \\
2 \mathrm{~K} \times 2 \mathrm{~K} \text { using } 5.0 \mu \mathrm{m} \text { cutoff } \mathrm{HgCdTe}\end{array}$ \\
\hline Redundancy & Fully redundant opto-mechanical design $\mathrm{w} /$ cross-strapped electronics \\
\hline
\end{tabular}

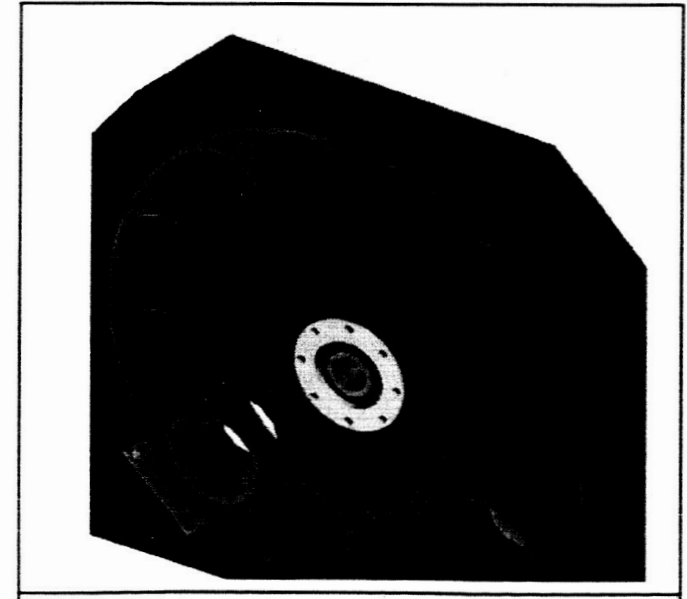

Figure 3: NIRCam filter wheel.

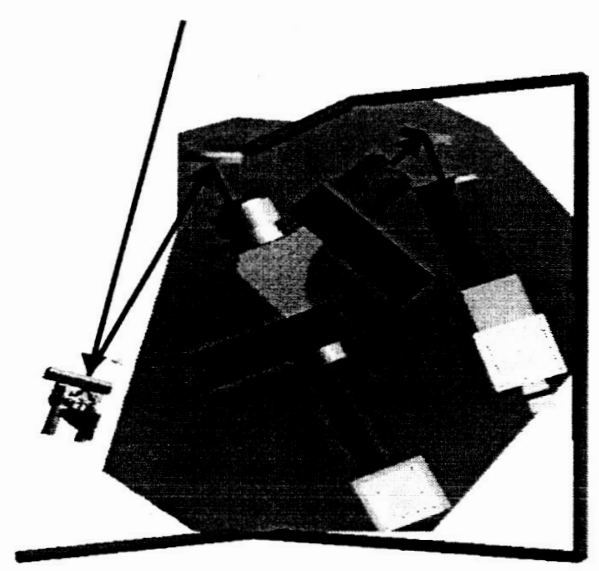

Figure 4: One half of NIRCam showing strut arrangement for mounting to ISIM. 


\subsection{The Mid-Infrared Instrument (MIRI)}

MIRI will play a crucial role in enabling the JWST science mission by obtaining images and spectra from 5 to $28 \mu \mathrm{m}$. The discovery of first light objects, for example, cannot be adequately performed without it. Figure 5 shows a plot of the expected brightness of such objects through 6 spectral bands in the near and mid-infrared; the 4 shorter bands are in the near-infrared instrument, NIRCam, the other two are in MIRI. At the shorter wavelengths the relative brightness among the bands are almost indistinguishable for true first light objects, older galaxies, and quasars. However, at 5.6 and 7.7 $\mu \mathrm{m}$, the colors of first light objects diverge from the others, and thus they can be properly identified.

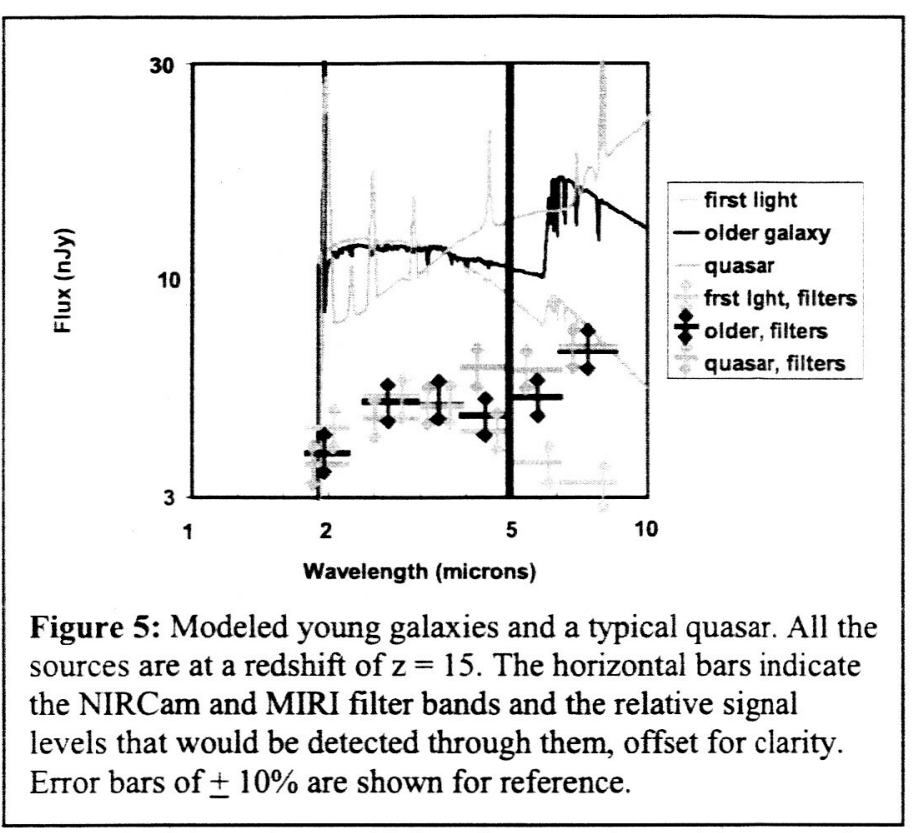

one hundred times the sensitivity of any previous telescope and instrument combination $(0.5 \mu \mathrm{Jy}$ at $10 \mu \mathrm{m}$ in a $3 \mathrm{hr}$ exposure).

MIRI (Figure 6) is a combination imager and spectrograph. It has 4 science modes summarized in Table 2:
In addition to key roles in the JWST defining science programs, the huge discovery potential of MIRI makes it most exciting: it has approximately

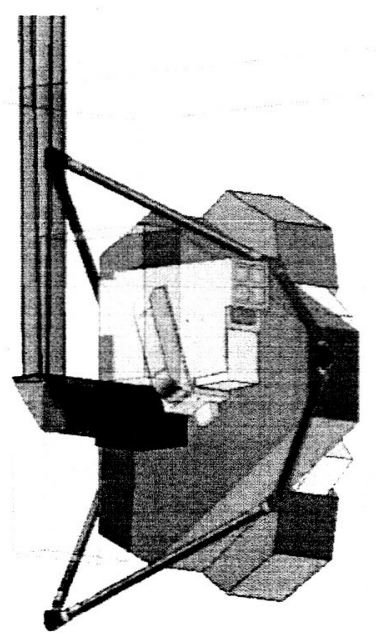

Figure 6: The MIRI optical bench assembly shown in its ISIM control volume.

\begin{tabular}{|l|l|l|l|l|}
\hline Table 2: MIRI Science Modes & Favelengths & $\begin{array}{l}\text { Spectral } \\
\text { Resolution }\end{array}$ & Field of View & Comments \\
\hline Mode & $\begin{array}{l}5.6,7.7,10,11.3, \\
12.8,15,18,21, \\
\text { and } 25.5 \mu \mathrm{m}\end{array}$ & $\mathrm{R}=5$ typical & $1.9 \times 1.4$ arcmin & $\begin{array}{l}\text { Broad- and } \\
\text { narrow-band } \\
\text { filters }\end{array}$ \\
\hline Imaging & $\begin{array}{l}10.65,11.3,16, \\
\text { and } 24 \mu \mathrm{m}\end{array}$ & $\mathrm{R}=10$ & $25 \times 25$ arcsec & $\begin{array}{l}\text { Lyot and } \\
\text { phase mask }\end{array}$ \\
\hline $\begin{array}{l}\text { Low-resolution } \\
\text { spectroscopy }\end{array}$ & $5-11 \mu \mathrm{m}$ & $\mathrm{R}=100$ & $5 \times 0.2$ arcsec slit & \\
\hline $\begin{array}{l}\text { Medium-resolution } \\
\text { spectroscopy }\end{array}$ & $5-28.5 \mu \mathrm{m}$ & $\mathrm{R}-2000$ & $3.5 \times 3.5$ to $7 \times 7$ arcsec & $\begin{array}{l}\text { Integral } \\
\text { field unit }\end{array}$ \\
\hline
\end{tabular}

The scientific priorities and capabilities of MIRI are set by a team of astronomers from the United States and Europe. The MIRI itself is being built as a " $50 / 50$ " partnership between NASA and the European Space Agency, implemented at the Jet Propulsion Laboratory (Pasadena, CA) and by a consortium of astronomical and technical institutes in 10 
European nations including the UK, Netherlands, France, Germany, Sweden, Belgium, Spain, Denmark, Ireland, and Switzerland

MIRI will be delivered to the JWST project (at the Goddard Space Flight Center) for integration into the telescope system in 2009, and JWST will be launched in 2011. For further information, see: http://ircamera.as.arizona.edu/MIRI and G. Wright, et al.: paper 5487-28, of these proceedings.

\subsection{The Near-Infrared Spectrograph (NIRSpec)}

NIRSpec is a state-of-the-art near-infrared multi-object spectrograph, designed to be capable of obtaining the near-IR spectra of 100 or more astronomical sources simultaneously at a spectral resolution of $R \sim 100$ over the $0.6-5$ micron wavelength range, or at a spectral resolution of $\mathrm{R} \sim 1000$ over 1 - 5 micron. The $\mathrm{R} \sim 100$ mode employs a single prism as its dispersive element and is intended for measuring the redshifts and continua spectra of faint galaxies. The $\mathrm{R} \sim 1000$ mode utilizes three diffraction gratings to cover the $1-5$ micron spectral region, and is primarily intended for detailed follow-up observations using conventional nebular emission lines as astrophysical diagnostics. Lastly, three $\mathrm{R} \sim 3000$ gratings also covering $1-5$ micron will allow kinematic studies of individual galaxies to be carried out in single object mode.

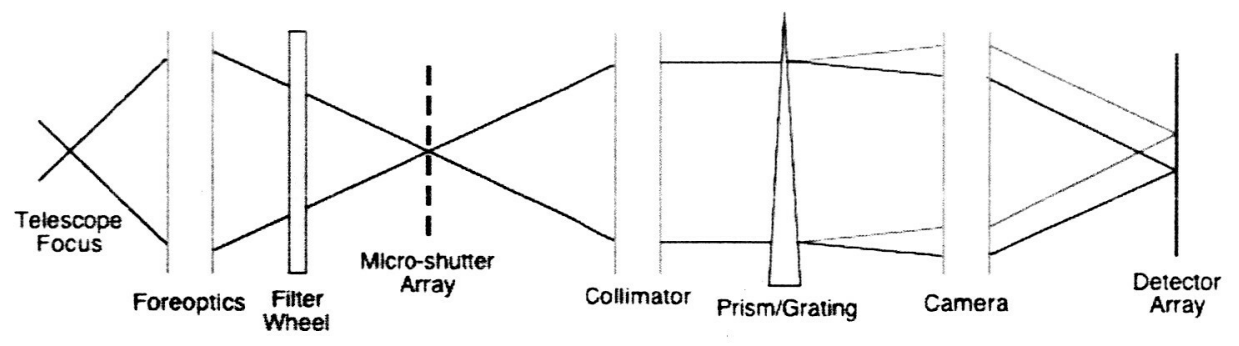

Figure 7: Schematic of the NIRSpec optical system.

The NIRSpec design employs all reflective optics, with most of its optical and structural elements manufactured out of modern ceramic materials ( $\mathrm{SiC}$ and/or C-SiC). The optical chain (Figure 7) has three main components. The fore-optics re-image and magnify the focal plane image of the JWST telescope proper onto the slit selection mechanism. The collimator converts the light emerging from each slit into a parallel beam and projects it onto the grating wheel which carries the six (flat) reflective gratings, the (dual pass reflective) prism and a mirror flat for target acquisition. The camera finally focuses the dispersed collimated light coming off the grating onto the detector array. A filter wheel located in an internal pupil of the fore-optics carries the requisite order separation filters for the diffraction gratings and also serves as the instrument shutter

The NIRSpec slit selection mechanism (Figure 8) is a programmable Micro Shutter Array (MSA) to be provided by Goddard Space Flight Center (GSFC). The MSA is made up of four $384 \times 175$ sub-arrays of individually programmable shutters. The open area of each shutter is 200 mas wide and 450 mas long. The active area of the whole MSA spans a field of view measuring 9 square arc-minutes on the sky. In addition to the programmable micro shutters, the MSA also carries several fixed slits that can be used for high contrast observations of single objects at any of the three spectral resolutions. Resources permitting, a passive small field ( 3" x 3") Integral Field Unit (IFU) may also be included for

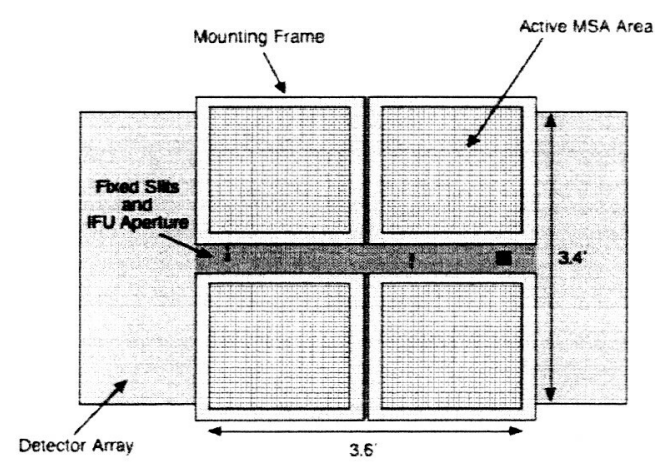

Figure 8: Schematic layout of the NIRSpec slit mask overlaid the detector array. use with the three $R \sim 3000$ gratings. 
The NIRSpec detector system, also provided by GSFC, consists of two close-butted $2 \mathrm{k}$ x $2 \mathrm{k}$ Rockwell HgCdTe detector arrays. In order to optimize the detector noise limited faint-end sensitivity of NIRSpec, the detector samples the spectra at a relatively coarse 100 mas per pixel.

NIRSpec is expected to be capable of reaching continuum fluxes approaching $\sim 125 \mathrm{nJy}$ at $\mathrm{S} / \mathrm{N} \sim 10$ in $\mathrm{t} \sim 10^{4} \mathrm{~s}$ in $\mathrm{R} \sim 100$ mode, and line fluxes as faint as $\sim 5 \times 10^{-19} \mathrm{erg} \mathrm{s}^{-1} \mathrm{~cm}^{-2}$ at $\mathrm{S} / \mathrm{N} \sim 10$ in $\mathrm{t} \sim 10^{5} \mathrm{~s}$ in $\mathrm{R} \sim 1000$ mode.

NIRSpec will be built in European industry under ESA leadership and funding. The prime contractor for NIRSpec is presently in the process of being selected and will be announced in late June 2004. For further information see F. Zamkotsian, et al.: paper 5487-26 and H. Moseley, et al.: paper 5487-27, of these proceedings.

\subsection{The Fine Guidance Sensor (FGS) and Tunable Filter Instrument}

The FGS provides guiding for the JWST and also tunable narrow-band filter science imaging capability. These two functions are named FGS-guider and FGS-TF. They have separate fields of view and also separate optical and detector systems. Figure 9 shows how they are laid out on two sides of the optical bench.

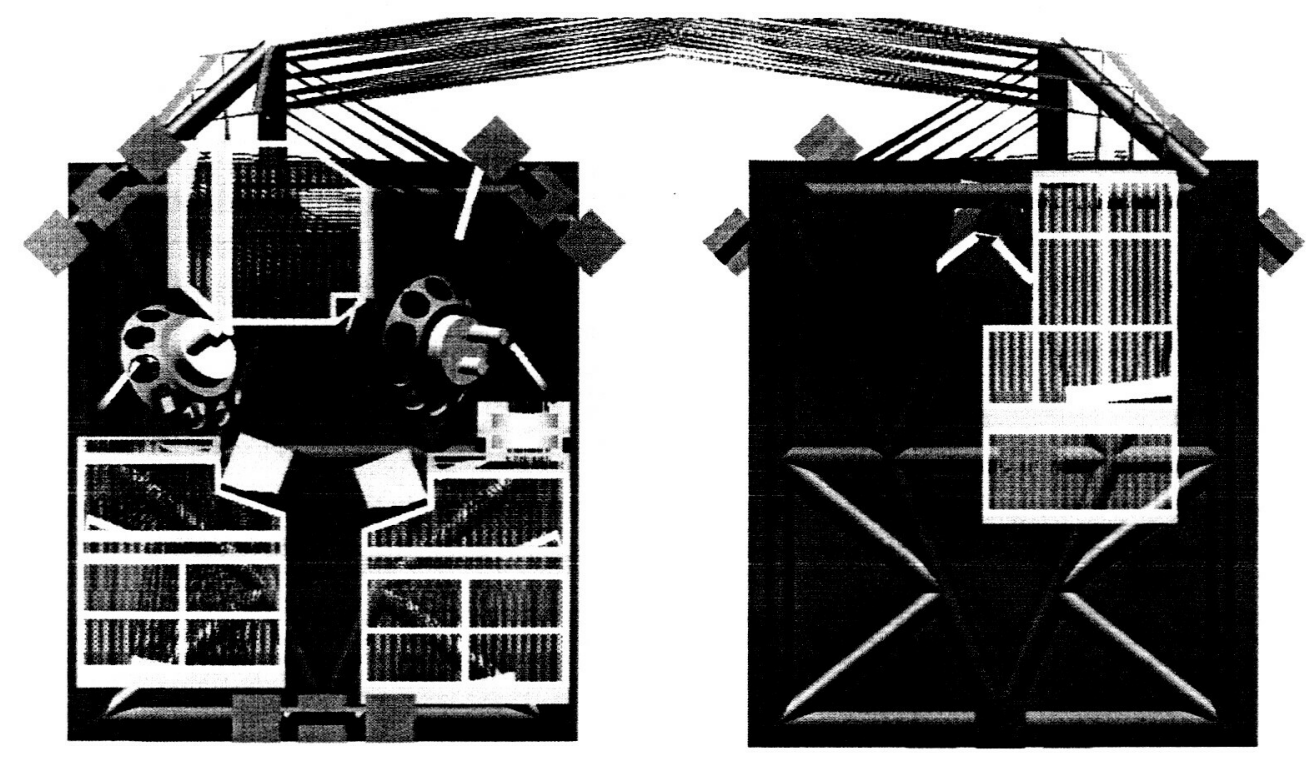

Figure 9: FGS guider side (left) and FGS-Tunable Filter side (right), with ray traces. Yellow boxes contain optics modules.

The FGS consists of an optical assembly, a set of Focal Plane / Instrument Control Electronics and two Command \& Data Handling Units. The whole instrument fits in the ISIM in a single box, with two pickoff mirrors. The FGS communicates directly with the spacecraft ACS because of its guiding function.

The optical assembly of the FGS instrument (Figure 9) consists of two modules each with two channels. The guider module images two separate regions of the sky on to two independent $2 \mathrm{k} \times 2 \mathrm{k}$ focal plane arrays. The TF module consists of a single field of view that is simultaneously imaged by two separate $2 \mathrm{k} \times 2 \mathrm{k}$ focal plane arrays in two different wavelength regions, via a beam-splitter. Each TF channel contains a filter wheel and tunable filter that allow flexible narrow-band imaging to be performed in the two wavelength bands simultaneously. 
The guider has two adjacent fields of $2.3^{\prime}$ on a side, with 68 mas pixels. These will enable the required guiding precision of 4.9 mas at $16 \mathrm{~Hz}$, with a faint limit that gives $95 \%$ guide star probability in the emptiest part of the sky, with full redundancy. Guiding on moving targets is also a potential capability. The two detectors are fed by a single set of relay optics, and operate over wavelengths 0.8 to 2.5 microns.

The TF side has the same field of view and pixel size (and can also be used for guiding). The beam splitter allows the field to be viewed with filters approximately in the 1.1-2.4 and 2.4-5.0

micron ranges, simultaneously. The filters can be tuned within this range with a spectral resolution of about 100 , and scanning can

yield spatially resolved spectra of all objects in the field of view. There are also coronagraph spots at the edge of the field that provide central light suppression of $10^{-5}$ at 1 arcsec radius.

For further information, see N. Rowlands, et al.: paper 5487-29, of these proceedings.

\section{SCIENCE SUPPORT SYSTEMS}

\subsection{Optical Metering Structure Subsystem}

The Optical Metering Structure (Structure) shown in Figure 10 houses the Science Instruments (SIs), providing structural support for ground testing and launch, and maintaining the correct position of the science instruments with respect to each other and the Optical Telescope Element (OTE).

The Structure is a three-dimensional frame made from M55J/954-6 Graphite Fiber Reinforced Plastic (GFRP) square tubes, held

together with GFRP gussets and clips, and with metal node fittings at a few select locations (Figure 11). The tube topology is driven by OTE and SI accommodation, i.e., the need for stable attachment between the ISIM and OTE near the primary mirror, need for stable attachment between ISIM and the SIs near the light coming from OTE, and the limits of physical space available within the overall JWST architecture. The SIs and OTE will attach to ISIM via flexural mounts to provide a nearly statically determinant interface and minimize the effects of differential Coefficient of Thermal Expansion (CTE) between the SIs, the Structure and the OTE. GFRP was selected because of its high specific stiffness and near-zero CTE at the operating temperature, and preliminary design analysis

\begin{tabular}{|ll|}
\hline Payload mass & $970 \mathrm{~kg}$ \\
Structure mass & $300 \mathrm{~kg}$ \\
OTE Flexure mass & $30 \mathrm{~kg}$ \\
& \\
Funcamental Frequency & $>25 \mathrm{~Hz}$ \\
Gravity distortion & $<0.5 \mathrm{~mm},<2$ arcmin \\
& \\
On-Station Temperature & $32-37 \mathrm{~K},<2 \mathrm{~K}$ gradient \\
Cooldown distortion (ambient to on-station) & $<1 \mathrm{~mm},<3$ arcmin \\
Dimensional Stability (0.5 K bulk change) & $<0.0002 \mathrm{~mm},<0.01$ arcmin \\
\hline
\end{tabular}

Figure 12: Overview of Key Requirements indicates the Structure can meet the challenging mass, stiffness, and thermal distortion requirements (Figure 12). 
The Structure requirements are believed to be pushing the state of the art in GFRP performance. Two specific challenges are insufficient material properties data at cryogenic temperatures needed for design analysis, and the difficulty in analyzing the thermal distortion of large, complex structures to the accuracies required. To address these needs, the Structure team is executing a comprehensive program to characterize and model the thermal distortion of the constituent materials and structural elements at successively higher levels of assembly, starting with GFRP lamina/laminates, adhesives, and metals, proceeding through tubes and partial frames, and concluding with the complete Structure and integrated ISIM.

The Structure design includes metal fittings bonded into the GFRP tubes at select locations. The baseline fitting is Titanium 6Al-4V ELI, a high strength aerospace material suitable for cryogenic applications. The large differential thermal strain between Titanium and GFRP raises concerns about survivability of bonded metal/composite joints when exposed to cryogenic temperatures, and the durability of the joints after repeated exposures to cryogenic temperatures during ground testing of the ISIM and the integrated ISIM/OTE prior to launch. To address these concerns, the Structure team is executing a plan to demonstrate survivability and durability with sufficient margin by developing, analyzing and testing metal/composite joints of increasing complexity and sophistication, starting with simple flat coupons, moving to tubes/fittings, and culminating with testing of the complete Structure.

The Structure team is also working to understand other critical aspects of GFRP performance in this application, including: stress relaxation over time; microcrack formation and its effects on dimensional stability over repeated thermal cycles; radiation effects on various properties; elastic properties from room temperature to cryogenic temperatures; degree of cure and its effects on various properties; moisture absorption/desorption from room temperature to cryogenic temperatures and its effects on dimensional stability and molecular contamination.

\subsection{Passive Cryogenic Thermal Control Subsystem}

Near-infrared Detectors, which form the heart of three of four instruments on the ISIM platform, must be cooled by the ISIM Thermal Control Subsystem to $37 \mathrm{~K}$ to provide the required sensitivity for scientific observations. The entire ISIM platform structure must also be cooled to approximately this temperature and, to provide required repeatability and alignment control for the instrument optics, must be kept thermally stable to within $0.5 \mathrm{~K}$ and $1 \mathrm{~K}$ over a 10000 second, and 24 hour period, respectively. The ISIM Thermal Control Subsystem must provide passive thermal control of the ISIM and its scientific instrument payload at cryogenic temperatures while in close proximity to the spacecraft and other instrument and detector electronics at near ambient temperatures. The difficulty of this task lies in part in the balancing of the direct and parasitic heat loads with the heat rejection capabilities of available cryogenic radiators to achieve the required temperature with the mandated $50 \%$ predicted thermal margin until at least the CDR.

Further complicating the thermal control is
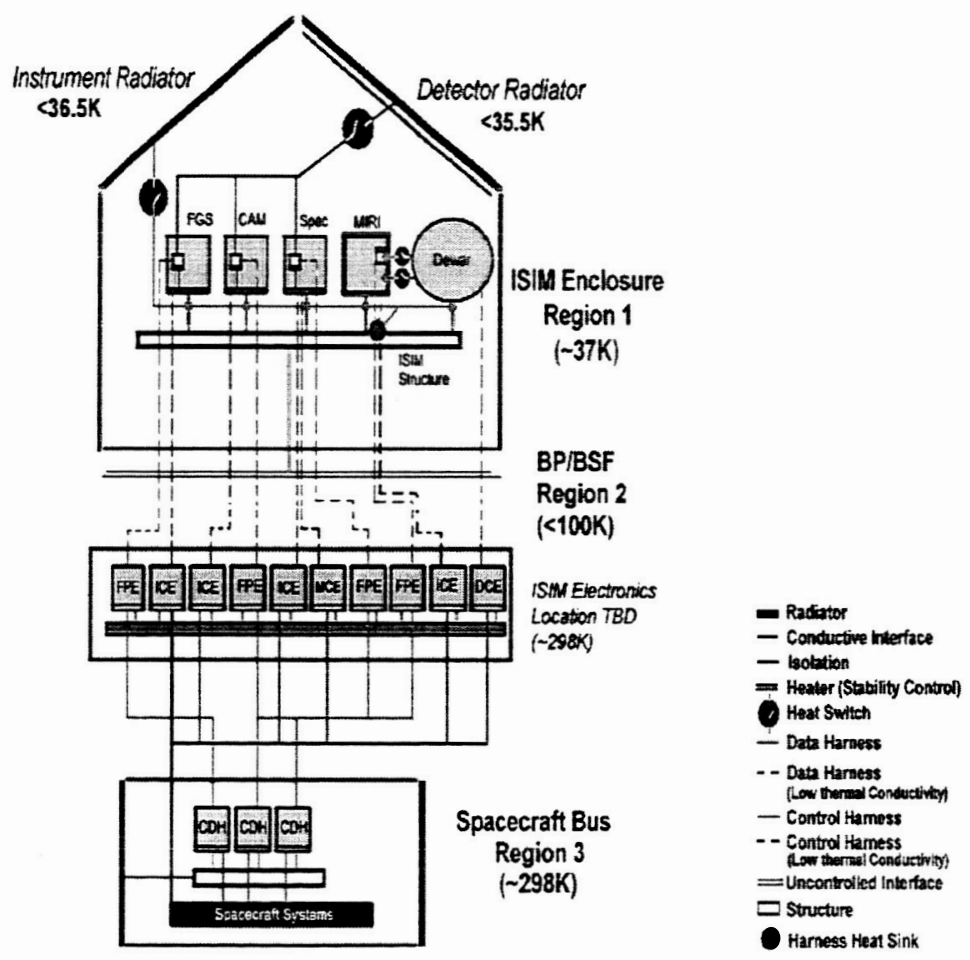

Fiaure 13: Schematic of ISIM Thermal Control 
the fact that the ISIM platform structure, being a carbon-based composite, is the potential source of substantial entrapped water. Careful thermal control of the instruments and detectors during the initial post-launch cool-down is required to avoid moisture contamination of instrument detectors and sensitive optics. Practical considerations require that instrument conductive coupling to the radiators be controllable to allow the instruments and their detectors to be warmed to approximately $165 \mathrm{~K}$ until the structure cools below $140 \mathrm{~K}$, permanently entrapping the moisture within it or on nonsensitive surfaces.

The 37K ISIM and its science instrument payload (designated "region 1") lie on the anti-sun side of the large sunshields, permanently shaded from the sun and the warm bus and solar panels. Milliwatt-level heat load allocations have been assigned to each of the instruments and housekeeping electronics circuits situated within this region. Two cryogenic radiators, one dedicated to the near-infrared detectors, and one dedicated to their associated science instrument optics assemblies and ISIM structure, are used to cool Region 1 to its required operational temperature range (Figure 13). Extensive tests of suitable candidate radiator coatings and analytical simulations are being performed to obtain the optimal radiator coating. High purity aluminum heat straps will be used to couple the three near-infrared science instruments and their detectors to their respective radiators with minimal temperature drop.

The need for active control of the detectors and science instruments during cool-down to prevent moisture contamination onto sensitive surfaces requires the ability to temporarily decouple the instruments and their detectors from their respective radiators while controlled heat is applied. This decoupling is accomplished by use of novel heat switches, which use the principle of differential thermal expansion of internal components to "Close" or "Open" a thermal circuit on command. When closed, during normal instrument operation, these switches are completely passive. Opening them (only done to prevent contamination, while the instruments are not taking scientific data) requires a small amount of added heat. Research and development is currently being performed to provide heat switches with the proper operational characteristics and reliability for the JWST mission.

\subsection{Command and Data Handling Subsystem}

The ISIM Command and Data Handling (ICDH), is a subsystem of the ISIM (Figure 14). The ICDH hardware and software together provide three major functions:

1. Coordinate simultaneous collection of science image data from the NIRCam, NIRSpec and MIRI instruments in support of parallel science objectives

2. Perform multi-accum \& lossless compression algorithms on science image data to achieve data volume reduction needs for on-board storage and subsequent transmission to the ground

3. Communicate with the Spacecraft Command and Telemetry Processor (CTP) to transfer data to the Solid State Recorder (SSR) and prepare for subsequent science operations

An overview of the JWST Observatory highlighting the ISIM electrical interfaces is shown in Figure 14.

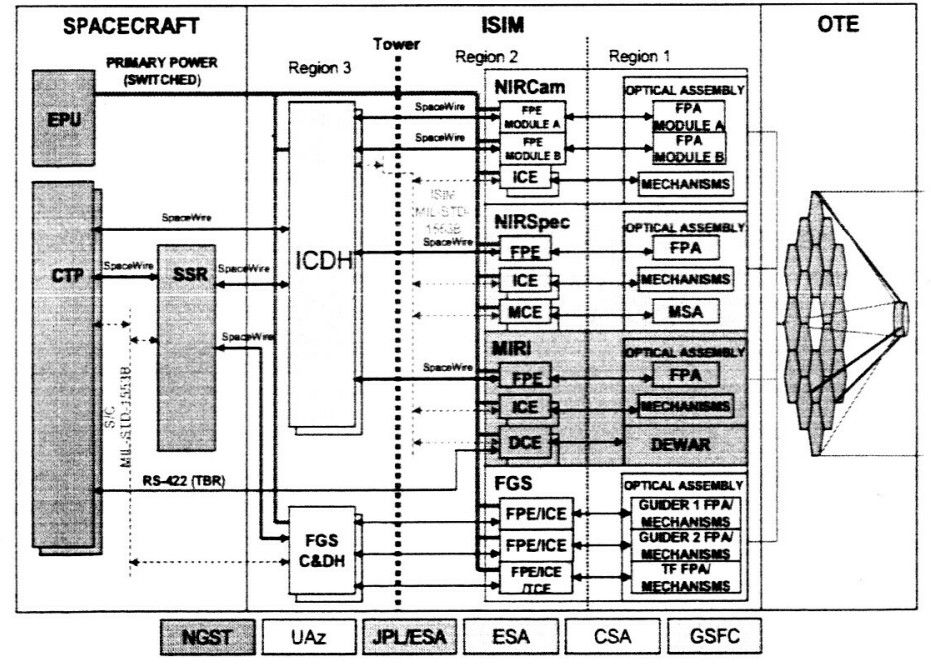

Figure 14: ISIM Electrical Interface Diagram

The ICDH hardware consisting of 7 types of assemblies is shown in Figure 15 and the general science image data collection, processing and storage flow is shown in Figure 16. 


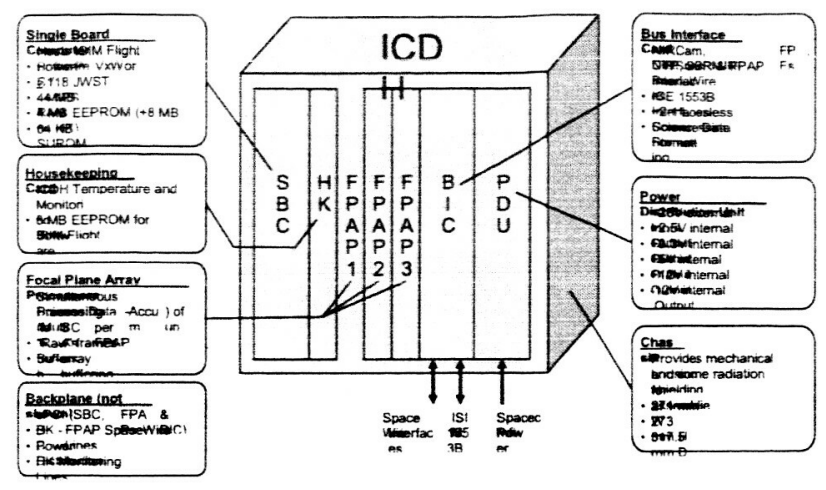

Figure 15: ICDH Assembly

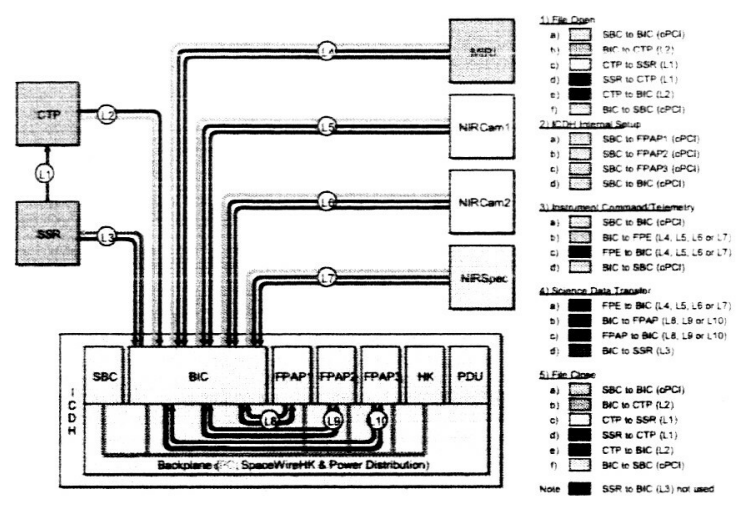

Figure 16: Science Image Data Flow Diagram

Table 3: Spacewire Interface Descriptions

\begin{tabular}{|c|c|c|c|c|c|c|c|c|}
\hline Link & Link Direction & Packet Type(s) & $\begin{array}{l}\text { Assigned Link } \\
\text { Rate* (Mbps) }^{*}\end{array}$ & $\begin{array}{c}\text { Max FCT } \\
\text { Cost }^{*} \text { (Mbps) }\end{array}$ & $\begin{array}{l}\text { Effective Link } \\
\text { Rate (Mbps) }\end{array}$ & $\begin{array}{l}\text { Max Pkt Data } \\
\text { Rate (Mbps) }\end{array}$ & $\begin{array}{l}\text { Needed Link } \\
\text { Rate (Mbps) }\end{array}$ & Margin \\
\hline \multirow{2}{*}{$\mathrm{L} 1$} & CTP to SSR & Command & 10.0 & -1.0 & 9.0 & 0.1 & 0.1 & $7100 \%$ \\
\hline & SSR to CTP & Science \& Telemetry & 20.0 & -0.5 & 19.5 & 8.0 & 10.0 & $95 \%$ \\
\hline \multirow{2}{*}{ L2 } & $B I C$ to CTP & Command \& Teiemetry & 10.0 & -0.5 & 9.5 & 0.3 & 0.4 & $2433 \%$ \\
\hline & CTP to BIC & Command \& Telemetry & 10.0 & -0.5 & 9.5 & 0.3 & 0.4 & $2433 \%$ \\
\hline \multirow{3}{*}{ L3 } & & Uncompressed Image & \multirow{2}{*}{48.0} & \multirow{2}{*}{0.0} & 48.0 & 23.2 & 29.0 & $66 \%$ \\
\hline & BIC to SSR & 2:1 Compressed Image & & & 48.0 & 11.6 & 14.5 & $231 \%$ \\
\hline & SSR to BIC & $n / a$ & 10.0 & -2.4 & 7.6 & nia & $\mathrm{n} / \mathrm{a}$ & n/a \\
\hline \multirow{3}{*}{ L4 } & BIC to MIRI & Command & 10.0 & -1.7 & 8.4 & 0.1 & 0.1 & $6580 \%$ \\
\hline & \multirow{2}{*}{ MIRI to BIC } & Pixel & \multirow{2}{*}{33.0} & \multirow{2}{*}{-0.5} & \multirow{2}{*}{32.5} & 20.6 & 25.8 & \multirow{2}{*}{$21 \%$} \\
\hline & & Telemetry & & & & 0.8 & 1.0 & \\
\hline \multirow{3}{*}{ L5 } & BIC to NIRCam1 & Command & 10.0 & -3.3 & 6.7 & 0.1 & 0.1 & $5260 \%$ \\
\hline & \multirow{2}{*}{ NIRCam 1 to BIC } & Pixel & \multirow{2}{*}{66.0} & \multirow{2}{*}{-0.5} & \multirow{2}{*}{65.5} & 34.4 & 43.0 & \multirow{2}{*}{$49 \%$} \\
\hline & & Telemetry & & & & 0.8 & 1.0 & \\
\hline \multirow{3}{*}{16} & BIC to NIRCam2 & Command & 10.0 & -3.3 & 6.7 & 0.1 & 0.1 & $5260 \%$ \\
\hline & \multirow{2}{*}{ NIRCam2 to BIC } & Pixel & \multirow{2}{*}{66.0} & \multirow{2}{*}{-0.5} & \multirow{2}{*}{65.5} & 34.4 & 43.0 & \multirow{2}{*}{$49 \%$} \\
\hline & & Telemetry & & & & 0.8 & 1.0 & \\
\hline \multirow{3}{*}{17} & BIC to NIRSpec & Command & 10.0 & -1.7 & 8.4 & 0.1 & 0.1 & $6580 \%$ \\
\hline & \multirow{2}{*}{ NIRSpec to BIC } & Pixel & \multirow{2}{*}{33.0} & \multirow{2}{*}{-0.5} & \multirow{2}{*}{32.5} & 13.8 & 17.2 & \multirow{2}{*}{$79 \%$} \\
\hline & & Telemetry & & & & 0.8 & 1.0 & \\
\hline \multirow{2}{*}{ L8 } & BIC to FPAP1 & Pixel & 96.0 & -4.8 & 91.2 & 41.3 & 51.6 & $77 \%$ \\
\hline & FPAP1 to BIC & Processed Image & 96.0 & -4.8 & 91.2 & 36.4 & 45.5 & $100 \%$ \\
\hline \multirow{2}{*}{ L9 } & BIC to FPAP2 & Pixel & 96.0 & -4.8 & 91.2 & 41.3 & 51.6 & $77 \%$ \\
\hline & FPAP2 to BIC & Processed Image & 96.0 & -4.8 & 91.2 & 36.4 & 45.5 & $100 \%$ \\
\hline 10 & BIC to FPAP3 & Pixel & 96.0 & -4.8 & 91.2 & 41.3 & 51.6 & $77 \%$ \\
\hline L10 & FPAP3 to BIC & Processed Image & 96.0 & -4.8 & 91.2 & 36.4 & 45.5 & $100 \%$ \\
\hline
\end{tabular}

Notes: * Run-time link rates shown. All links (re-)initialize at $10 \mathrm{Mbps}$.

- 6.4 Mbps raw data per detector: 16 bit pixels $x 4$ pixels / 10 usec

- $8.6 \mathrm{Mbps}$ science packets per detector: (( 16 bits per pixels $\times 16$ pixels +2 header bytes $) \times 10$ SpaceWire bits +4 bit EOP $) / 40$ usec

- 1 FCT (4-bits) for every 8 bytes ( 80 bits encoded) in opposite direction

- Max active detectors assumed: 3 for MIRI, 5 for NIRCam1, 5 for NIRCam2 \& 2 for NIRSpec

The ICDH communicates with the Instruments over Spacewire Point-to-Point interfaces operating at link rates between $10 \mathrm{Mbps}$ to $66 \mathrm{Mbps}$. The internal Spacewire communications occur between the BIC to FPAP assemblies at a link rate of $96 \mathrm{Mbps}$ as shown in Table 3.

The ICDH provides the centralized ISIM Command and Data Handling functions for the NIRCam, NIRSpec, and MIRI instruments and unifies the Image collection with a common Spacewire point to point topology. The JWST ICDH is unique in that it centralizes the C\&DH functions for NIRCam, NIRSpec, and MIRI away from the detector electronics region in order to manage stringent thermal requirements while maintaining the ability to receive image data at peak detector readout rates. A Spacewire ASIC with a 12 port Router enables the High Speed Extraction of Image data from 
each of the instruments and routes the data to each of the Focal Plane Array Processors (FPAP). The FPAPs are unique in that they are dynamically configurable and assignable to any one of the 15 total NIRCam. NIRSpec, and MIRI instrument Sensor Chip Assemblies. The ICDH interfaces to each of the Instruments allows for unique and independent science operations while providing a standard protocol across the interface ultimately resulting in integration and test risk reduction at the ISIM Level. Finally the ICDH has been architected to meet high data throughput and processing requirements while minimizing overall lifecycle costs and risks.

\subsection{Flight Software Subsystem}

The ISIM flight software (FSW) can be divided into four basic categories: the Core Command and Data Handling (CC\&DH) software, ISIM general support software, ISIM instrument ${ }^{1}$-specific software, and interface control software for communication to various external devices. This architecture is shown schematically in Figure 17 . Infusing the whole is the Real-time Object Oriented Methodology (ROOM) used for its creation. We will discuss it first, and then move on to the functionality of each of the ISIM FSW elements.

The ISIM FSW is being developed using the ROOM paradigm as realized via IBM's Rose Real-Time development tool. It is proving to be a powerful, yet simple, paradigm - particularly as realized via Rose Real Time's graphical interface. Being object-oriented, the fundamental building blocks are classes - although with a bit of a twist. There are three class types: passive classes (a.k.a. "classes"), active classes (a.k.a. "capsules"), and protocol classes (a.k.a. "protocols"). Passive classes are the standard classes of basic object orientation; they contain attributes (i.e., variables in the form of primitive types or instances of other passive classes) and methods (i.e., class-specific functions). Capsules are more powerful constructs than passive classes. In

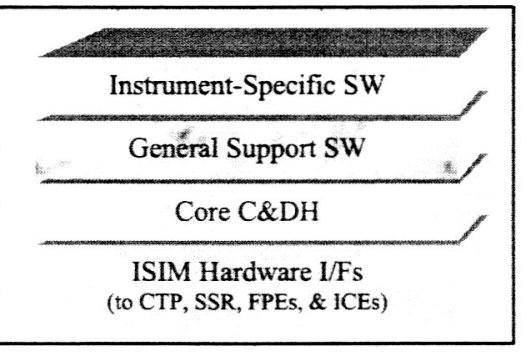

Figure 17: - ISIM FSW Categories addition to attributes and methods, each capsule contains some number of ports for communicating with other capsules and a state machine that defines the capsule's action upon receiving signals from other capsules. Each port is an instance of a corresponding protocol, which defines a set of permitted incoming and outgoing signals. The data content of a given signal is typically defined in terms of some passive class, although it can be left empty. A capsule's state machine consists of at least one state, but may be composed of a fairly complex set of states and transition relationships between them. Receipt of a signal via a port triggers a state-to-state transition (possibly from a state into itself), with said transition consisting of a set of code lines to be executed in response to the signal. This transition code may include invocation of one or more port-defined outgoing signal methods, thereby allowing the capsule to communicate to other capsules. The net result is that receipt of a signal can cause a capsule to change its state, change its attribute values, and send out response signals all as a function of the content of the received signal.

To date, the bulk of the effort for ISIM FSW development has been devoted to the CC\&DH, which is designed to be a generic and highly reusable FSW infrastructure package. Now close to complete, the CC\&DH consists of the following tasks, each realized as a capsule instance.

- Command Management: delivers command signals from one capsule to another. Upon startup, each task (capsule) registers its unique ID with command management, which thereafter knows how to deliver messages to it.

- Telemetry Management: delivers each telemetry packet generated by a task to any other task that has registered to receive packets of that type. Telemetry management also coordinates delivery of the telemetry packets to the solid state recorder and to the real-time telemetry stream.

- Time Management: converts the spacecraft oscillator's time tick to a Universal Time Coordinated (UTC) time packet and delivers the packet to all tasks that registered to receive it.

- Table Load and Dump: provides a unified mechanism for loading and dumping task parameter tables.

- File Management: manages onboard files, in particular those used to support the mission observation plan. This includes providing a file listing mechanism, decompressing g-zipped versions of files, and deleting unneeded files.

${ }^{1}$ For programmatic reasons, the ISIM FSW does not directly interface with or control the FGS-Guider or FGS-TF modules. The term "science instrument" in this section is limited to the NIRCam, NIRSpec, and MIRI. 
- File Transfer: coordinates loading and dumping of files to RAM or ROM using CCSDS File Delivery Protocol (CFDP)

- Script Processing: provides a wrapper for a COTS JavaScript engine, and serves as the interface between said engine and various CC\&DH services, particularly command and telemetry management. Based on the current operations concept, normal mission activities (e.g., slewing, acquiring guide stars, acquiring targets, etc.) will be coordinated with scripts.

- Stored Commanding: provides for storage and issuance of absolute time and relative time command sequences. Based on the current operations concept, it is expected that this function will be used primarily for responding to anomalies.

- Subsystem Telemetry Monitoring: allows for the defining of telemetry variables to be monitored relative to various limit conditions, and specifies appropriate stored command sequences to be triggered in case of limit violations.

- Memory Management: provides functions for dumping the content of specified memory areas (both as a single snapshot and over time), performing CRC-based checks on memory content, and performing periodic volatile memory integrity checks using the hardware-supplied error detection and correction circuit

- Computer Health and Safety: monitors health and safety signals from both ISIM FSW tasks and the external systems (e.g., the spacecraft CTP) and initiates predefined actions in case of failures

- Executive Services: provides an interface to operating system (i.e., VxWorks) services.

In contrast to the CC\&DH, the remaining software elements are mission specific. This section covers two categories: ISIM general support, and ISIM instrument-specific software. The general support software provides coordination of the mission plan as a whole, and also provides utility functions needed by all of the science instruments. The category "instrument-specific software" pertains to those modules to be written by the SI teams with functionality unique to each

SI. The individual functions are:

- Observation Plan Executive: coordinates execution of the observation plan, feeding requests to the CC\&DHbased script processor with names of script files to process and associated parameter values to use as input.

- Exposure Support: transforms a "high-level" parameter set defining a science instrument exposure into commands to the Focal Plane Electronics (FPE), Focal Plane Array Processor (FPAP), and Bus Interface Card (BIC) to cause the acquisition of science data, and coordinates the writing of the data to the solid state recorder.

- Target Acquisition Support: provides utility functions (e.g., star image centroiding) for processing science instrument data acquired for the purpose of locating a science target and moving it to a selected position (e.g., a coronagraph occulting disk, or a spectrograph slit).

- NIRCam control and monitoring: instrument specific functions to be developed by SI team

- NIRSpec control and monitoring: instrument specific functions to be developed by SI team

- MIRI control and monitoring: instrument specific functions to be developed by SI team

The ISIM FSW also provides drivers for bus communications to various pieces of external hardware. This includes the interfaces to the spacecraft-based main processor (i.e., the CTP), the solid state recorder (SSR), the science instrument focal plane electronics (FPE) modules, and the instrument control electronics (ICE) modules.

\section{ACKNOWLEDGEMENTS}

We thank John Hutchings, Peter Jakobsen, Marcia Rieke, George Rieke, and Gillian Wright for science instrument descriptions and graphics. 